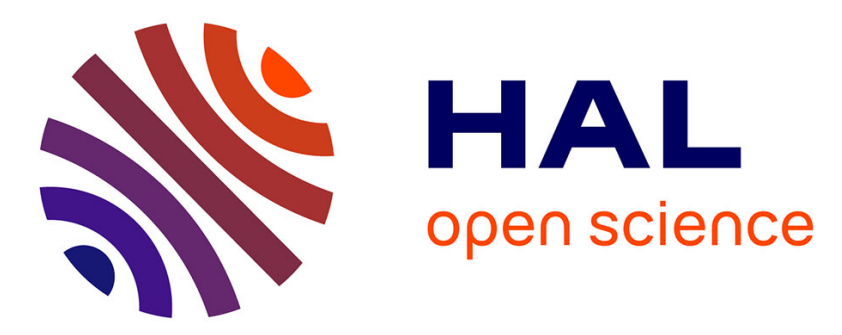

\title{
Energy migration effect on the formation mechanism of different unsaturations in ethylene/styrene random copolymers
}

\author{
M. Ferry, M. Ramillon, T. Been, P.J. Lutz, Y. Ngono-Ravache, E. Balanzat
}

\section{- To cite this version:}

M. Ferry, M. Ramillon, T. Been, P.J. Lutz, Y. Ngono-Ravache, et al.. Energy migration effect on the formation mechanism of different unsaturations in ethylene/styrene random copolymers. Polymer Degradation and Stability, 2019, 160, pp.210-217. 10.1016/j.polymdegradstab.2018.12.026 . hal02012021

\section{HAL Id: hal-02012021 \\ https://hal.science/hal-02012021}

Submitted on 19 Oct 2021

HAL is a multi-disciplinary open access archive for the deposit and dissemination of scientific research documents, whether they are published or not. The documents may come from teaching and research institutions in France or abroad, or from public or private research centers.
L'archive ouverte pluridisciplinaire HAL, est destinée au dépôt et à la diffusion de documents scientifiques de niveau recherche, publiés ou non, émanant des établissements d'enseignement et de recherche français ou étrangers, des laboratoires publics ou privés.

\section{(ㄷ)(1) $\$$}

Distributed under a Creative Commons Attribution - NonCommerciall 4.0 International 


\title{
Energy migration effect on the formation mechanism of different unsaturations in ethylene/styrene random copolymers
}

M. Ferry ${ }^{*}, 1$, J.-M. Ramillon ${ }^{1}$, T. Been ${ }^{1}$, P.J. Lutz ${ }^{* * 2}$, Y. Ngono-Ravache ${ }^{1}$ and E. Balanzat ${ }^{1}$

(1) CIMAP, UMR 6252, CEA-CNRS-ENSICAEN-UNICAEN, Bvd H. Becquerel, BP 5133, F-14070 Caen Cedex 5, France

(2) Université de Strasbourg, Institut Charles Sadron, CNRS UPR 22, 23 rue du Loess, BP 84047, 67034 Strasbourg Cedex 2, France

\begin{abstract}
To evaluate the influence of specific parameters, i.e. energy sink concentration and excitons and/or radical migration, on the mechanisms underlying the formation of double bonds in aliphatic polymers, materials with energy sinks in the chain were synthesized. By following the radiation-induced modifications in ethylene/styrene random copolymers, as a function of the styrene content and of the irradiation temperature, we were able to understand the formation mechanisms of trans-vinylenes, trans-trans-dienes, allyl radicals and vinyls. The irradiation temperature allows the discrimination between energy transfers (at $11 \mathrm{~K}$ and at room temperature, RT) and of radical migration (at RT). Irradiations were performed using swift heavy ions, and we could show that track overlapping has also an influence on the studied chemical group concentrations. For instance, trans-vinylenes are influenced by excitation transfer and radical migration: their formation decreases in presence of styrene aromatic rings, whatever the dose range and the irradiation temperature. On the opposite, vinyls are formed only at high ionizing density and are not influenced by excitation transfer; however, their concentration is influenced by radical migration.
\end{abstract}

\section{Keywords}

Ion irradiation; ethylene/styrene copolymers; FTIR; energy transfers; radical migration

\footnotetext{
* Corresponding author. E-mail: muriel.ferry@ @ea.fr. Phone: +(33)1-69-08-27-34

Now at : Den-Service d'Étude du Comportement des Radionucléides (SECR), CEA, Université ParisSaclay, F-91191, Gif-sur-Yvette, France

${ }^{* *}$ Retired
} 


\section{Introduction}

The hypothesis of energy transfers in organic molecules under irradiation was proposed during the early work of Schoepfle \& Fellows [1], who studied gaseous products emitted from different linear, cyclic and aromatic liquid hydrocarbons, irradiated under vacuum using Xrays. These authors have evidenced that a mixture of benzene and cyclohexane has a hydrogen radiation chemical yield lower than the one calculated from the law of averages.

Excitation and/or radical transfers have also been studied in different kind of polymers to evaluate their efficiency as a function of the localization of the acceptor and donor sites, but also as a function of their kind of migration: intra-chain $v s$ inter-chains. This kind of studies began thanks to the pioneering works of Charlesby [2] and Dole [3]. They deduced from the observations that transfers from one unit to another are efficient in random copolymers but not in block copolymers [3], which was attributed to a migration distance of a few repetition units. In a parallel way, migration was evidenced in copolymers but not in physical mixtures [4] which would indicate that migration only occurs along covalent bonds.

In the early 70's, a model of these transfers was realized by Partridge [5]. In this model,excitation transfers are very efficient: he estimated an excitation migration distance in a polyethylene of the order of $150 \mathrm{~nm}$ towards additives (triphenylamine and triphenylmethanol) [6]. At the end of the 70's, first reviews devoted to these energy transfers were published, describing more precisely the phenomena embedded in the mechanisms, as for instance those of Forster and Dexter [7]. More recently, we have initiated a systematic study of the impact of excitons and/or radical migration [8-11] on the radiation behavior of polymers and solid hydrocarbons. The term energy transfers used in this work is wide and includes the mass or reactive species transport (including radicals and ions), lone charge transfers (electrons and holes), and electronic excitation transfers. In the following, we will not differentiate electronic excitation transfers and lone charge transfers: they will be referred as excitation transfers. Mass or reactive species transport will be summarized as radical migration.

The energy sinks concentration is obviously a key parameter. The lower the concentration, the higher the effect per protection unit and the faster their degradation. We have already shown in a previous article [8], by irradiating ethylene/styrene copolymers at different temperatures, that the presence of styrene protects the ethylene moiety, at room temperature (RT) but also at $11 \mathrm{~K}$. At $11 \mathrm{~K}$, it has been supposed that almost all radicals are frozen and cannot migrate. By evaluating, on trans-vinylene formation, the effect of the 
irradiation temperature and of the styrene content, we showed that radiation protection phenomena are due to both types of transfers at least in the case of the formation of this chemical group. Nevertheless, this protection effect is more efficient at RT, proving that the radical transfers play also a role. Finally, the aromatic moiety protects the aliphatic one, but at its expense since the aromatic moiety is consumed [12].

Among new chemical groups created in polymers without heteroatom, under low ionizing radiations and under helium atmosphere, the most abundant are of the alkene type: transvinylene in polyethylene, vinylidenes in polypropylene, or vinyls in polybutene [13]. Under swift heavy ions, specific new bonds, such as alkynes or cumulenes, are also observed: these bonds are formed at low doses above a Linear Energy Transfer (LET) threshold. They are also formed below the LET threshold provided if materials are irradiated at very high doses. Hence, formation mechanism of each specific group depends of the ionization density, and can be differently affected by excitation and/or radical transfers.

This article is a complement to a previous paper [8]. The objective is now to evaluate on unsaturated groups other than trans-vinylenes, the effect of the presence of aromatic rings in the polymer. The effect of the irradiation temperature and of the styrene concentration in the polymer is here evaluated on four different chemical groups: trans-vinylenes (as a recall of the former article), allyl radicals, trans-trans-dienes and vinyls. The first three bonds are representative of the aliphatic moiety, whereas the case of vinyl is more ambiguous. As already presented, irradiations were performed at $11 \mathrm{~K}$, a temperature sufficiently low to suppose that only excitation transfers operate, and at RT, where excitation and radical migrations are allowed. We will show that the influence of transfers on $\mathrm{C}=\mathrm{C}$ formation are function of chemical structure of the polymer, of the irradiation temperature and of the dose deposited in the material.

\section{Experimental section}

\subsection{Ethylene/styrene random copolymer samples: PES}

Polyethylene chosen was a commercial PE from Atochem (PE ATO). Materials based on ethylene-styrene random copolymers have been specifically synthesized. The experimental procedure used to prepare and characterize the copolymers was fully explained in our previous article [8]. Summary of the different sample characterizations are given in Table 1. 


\begin{tabular}{|c|cccc|}
\cline { 2 - 5 } \multicolumn{1}{c|}{} & PE & PES 2.2 & PES 7.0 & PES 19.0 \\
\hline Styrene molar content $(\%)$ & - & 2.2 & 7.0 & 19.0 \\
Ethylene molar content $(\%)$ & 100 & 97.4 & 93.0 & 81.1 \\
\hline Crystallinity $(\%)$ & 88.0 & 43.2 & 19.9 & 2.9 \\
Melting temperature $\mathrm{T}_{\mathrm{m}}\left({ }^{\circ} \mathrm{C}\right)$ & 131 & 115 & 84 & 54 \\
Glass transition temperature $\mathrm{T}_{\mathrm{g}}\left({ }^{\circ} \mathrm{C}\right){ }^{*}$ & -128 & -133 & -145 & -155 \\
\hline Density $\left(\mathrm{g} . \mathrm{cm}^{-3}\right)$ & 0.950 & $0.944 \pm 0.004$ & $0.955 \pm 0.007$ & $1.012 \pm 0.011$ \\
\hline
\end{tabular}

Table 1: Polymer samples characteristics. ${ }^{*}: T_{g}$ is estimated.

To estimate the glass transition temperature $T_{g}$, we used the empirical relation (1) issued from the work of Lee and Knight [14], temperatures being expressed in Kelvin (K).

$$
T_{g}=0.36 \cdot T_{m}
$$

This simple empirical relation allows only a rough estimation of $\mathrm{T}_{\mathrm{g}}$, but the objective here was only to evaluate the state, i.e. glassy or rubbery, of our materials at RT. It is obvious that all polymers are in the glassy state at $11 \mathrm{~K}$, but the polymer's behavior at RT is a priori far less evident. Using relation (1), we can assess that, at ambient temperature, the non-crystalline phase of all polymers are in the rubbery state. It is deduced that the chain mobility is somehow similar for all materials irradiated at the same temperature.

Densities were determined by putting one polymer film in a solvent which has a specific mass higher than the one estimated for the material under consideration. Then, another solvent, with a specific mass lower than the estimated one, is added until the polymer is floating on the fence. At this point, the solvent mixture and the polymer have the same specific mass, this last being determined using relation (2), with 1 and 2 indexes as reference to the two solvents.

$$
\rho_{\text {polymer }}=\frac{m_{1}+m_{2}}{V_{1}+V_{2}}=\frac{m_{1}+m_{2}}{m_{1} / \rho_{1}+m_{2} / \rho_{2}}
$$

In these equations, $m_{i}$ is the mass of solvent $\mathrm{i}$ added in $\mathrm{g}, V_{i}$ is the volume $\mathrm{i}$ added in $\mathrm{cm}^{3}$ and $\rho_{i}$ is density of the solvent I added in $\mathrm{g} . \mathrm{cm}^{-3}$. The underlying hypotheses of this experiment are that the two solvents are totally miscible relative to each other, and that none of them solubilizes or even swells the polymer. We choose dichloromethane (Sigma-Aldrich reference 32222, $\rho_{1}=1.325 \mathrm{~g} . \mathrm{cm}^{-3}$ ) and hexane (Sigma-Aldrich reference 32293, $\rho_{2}=0.659$ g. $\mathrm{cm}^{-3}$ ). Each determination was triplicated and the error bar is defined as one $\sigma$ (standard deviation) of the triplicate. In the case of PE, density was given by the supplier. 


\subsection{Irradiation conditions}

Systems were irradiated using ${ }^{20} \mathrm{Ne}$ Swift Heavy Ion (SHI) beams. Ion beam irradiations were performed using the medium energy line facility of the GANIL accelerator, Caen, France. Irradiations were performed either at RT or at $11 \mathrm{~K}$, under vacuum, at normal incidence. A x-, y-scanned beam was used to ensure a homogeneous irradiation field over the sample surfaces (typically $0.75 \mathrm{~cm}^{2}$ ). The fluxes (Table 2) were chosen in order to limit the power deposition on the sample to $0.5 \mathrm{~mW} . \mathrm{cm}^{-2}$, thus avoiding any significant sample heating. The energy loss was calculated with SRIM, based on the TRIM code [15].

Polyethylene was purchased under the form of film. In the case of PES, samples were prepared by high pressure molding using a laboratory press. In any sample, the projectile range was by far much larger than the sample thickness (e). Elsewhere the beam energy was high enough to ensure a relatively constant LET over the sample thicknesses. In most cases, the relative decrease of the projectile energy in the sample was well below $20 \%$.

Materials studied are random copolymers of ethylene and styrene; there is thus an intimate mixture of both monomers moieties. As the ion beams used in this study are of high energy (>10 MeV.A $\mathrm{A}^{-1}$ ), secondary electrons ranges are larger than the monomers units. Considering different absorbed doses in both moieties does not make sense. Consequently for the absorbed dose calculation, the LET value used here is a mean value, taking into account the copolymer as a whole.

Errors on the values have been estimated. Statistical errors, which are for a given sample and for a single beam condition, are small. We can estimate that statistical errors of defect concentration are at most a few \%. The systematic errors are higher and are mainly due to sample thickness and dose errors. It is estimated to be equal to $10 \%$.

\subsection{Set-up}

The results presented here correspond to a Fourier Transform Infrared spectroscopy (FTIR) analysis of the radiation-induced modifications. FTIR spectra were acquired using a Nicolet巴 Magna 750 spectrometer, equipped with a MCT-A detector. They were acquired in the transmission mode, at a resolution of $2 \mathrm{~cm}^{-1}$, and with 128 accumulations to improve the signal to noise ratio. Interference fringes were avoided by recording the spectra in the presence of a polarized light, at Brewster angle $\left(55^{\circ}\right)$.

To record the in-film modifications, a specific device allowed us to irradiate and to record the FTIR spectra without removing the sample from the cell [16]. Consequently, the contact 
between the polymer and air, as well as the subsequent heating after low temperature irradiation, were avoided; the post-irradiation oxidation did not occur and no annealing precedes the sample analysis. This device is equipped with a cryogenerator, which allows a gradual temperature lowering down to $11 \mathrm{~K}$. The sample temperature is controlled by two sensors, a carbon resistance and a CLTS (Compound Linear Thermal Sensor). This sample irradiation temperature can vary from $11 \mathrm{~K}$ to RT. For direct comparison purposes, whatever the irradiation temperature, all FTIR spectra were recorded at $11 \mathrm{~K}$.

Irradiations at $11 \mathrm{~K}$ are performed under vacuum, at pressures below $10^{-7}$ mbar. Under these conditions, only the residual $\mathrm{H}_{2} \mathrm{O}$ molecules present in the irradiation cell tend to condense and accumulate on the sample surface. Characteristic infrared bands of water ices are found around $3400 \mathrm{~cm}^{-1}, 1600 \mathrm{~cm}^{-1}$ and $600 \mathrm{~cm}^{-1}$. Nevertheless, they do not interfere with bands used through the present study. They can be mathematically removed by spectra subtractions without any incidence on the resulting spectra because the ice is on top of the sample, without any influence in terms of Van der Walls interactions. 


\begin{tabular}{|c|c|c|c|c|c|c|c|c|c|c|}
\hline Name & $\begin{array}{l}\text { Styrene } \\
\text { content } \\
(\text { molar \%) }\end{array}$ & $\begin{array}{c}\text { Irradiation } \\
\text { temperature }(\mathrm{K})\end{array}$ & Projectile & $\begin{array}{c}\mathrm{E}_{\mathrm{i}} \\
\left(\mathrm{MeV} \cdot \mathrm{A}^{-1}\right)\end{array}$ & $\begin{array}{c}\mathrm{E}_{\mathrm{o}} \\
\left(\mathrm{MeV} \cdot \mathrm{A}^{-1}\right)\end{array}$ & $\begin{array}{c}\mathrm{LET} \\
\left(\mathrm{MeV} \cdot \mathrm{mg}^{-1} \cdot \mathrm{cm}^{2}\right)\end{array}$ & $\begin{array}{c}\mathrm{e} \\
(\mu \mathrm{m})\end{array}$ & $\begin{array}{c}\text { Flux } \\
\left(10^{8} \mathrm{~cm}^{2} \cdot \mathrm{s}^{-1}\right)\end{array}$ & $\begin{array}{l}\text { Fluence } \\
\left(10^{12} \mathrm{~cm}^{2}\right)\end{array}$ & $\begin{array}{c}\text { Maximum } \\
\text { dose (MGy) }\end{array}$ \\
\hline $\mathrm{PE}$ & 0 & \multirow{4}{*}{11} & \multirow{4}{*}{${ }^{20} \mathrm{Ne}$} & \multirow{4}{*}{13.6} & 13.2 & 3.9 & 22 & 5.1 & 18.3 & 11.3 \\
\hline PES 2.2 & 2.2 & & & & 13.0 & 3.9 & 45 & 5.1 & 10.9 & 6.8 \\
\hline PES 7.0 & 7.0 & & & & 12.5 & 3.9 & 60 & 5.1 & 11.0 & 6.8 \\
\hline PES 19.0 & 19.0 & & & & 12.6 & 3.8 & 55 & 5.2 & 11.2 & 6.8 \\
\hline $\mathrm{PE}$ & 0 & \multirow{4}{*}{300} & \multirow{4}{*}{${ }^{20} \mathrm{Ne}$} & \multirow{4}{*}{13.6} & 13.1 & 3.9 & 20 & 5.1 & 7.3 & 4.5 \\
\hline PES 2.2 & 2.2 & & & & 12.7 & 3.9 & 50 & 5.1 & 7.3 & 4.5 \\
\hline PES 7.0 & 7.0 & & & & 12.5 & 3.9 & 61 & 5.1 & 18.3 & 11.3 \\
\hline PES 19.0 & 19.0 & & & & 12.5 & 3.8 & 61 & 5.2 & 7.5 & 5.6 \\
\hline
\end{tabular}

Table 2: Irradiation conditions $\left(E_{i}\right.$ and $E_{o}$ are the energies of the projectiles at the entrance and at the exit of the films respectively; LET is the Linear Energy Transfer; $e$ is the sample thickness). PE: polyethylene and PES X: ethylene/styrene copolymers, with X the molar styrene content in the copolymer. 


\subsection{Data analysis}

The radiation-induced modifications appear in the FTIR spectra in two different ways. In the first one, infrared bands initially present in pristine samples, decrease or increase in intensity, with eventually tenuous changes in width and position. The second radiationinduced modification is the emergence of new bands.

In this article, we focus on different new bond formation: allyl radicals at $942 \mathrm{~cm}^{-1}$, transvinylenes at $967 \mathrm{~cm}^{-1}$, trans-trans-dienes at $985 \mathrm{~cm}^{-1}$ and vinyls at $1640 \mathrm{~cm}^{-1}$. Strictly speaking, the band at $1640 \mathrm{~cm}^{-1}$ refers to all asymmetric alkenes, that is to vinyls, but also to vinylidenes and cis-vinylenes. In this study, we observed neither vinylidenes nor cisvinylenes, these last bands being ruled out because of the absence of the corresponding $\omega_{=C H}$ (wagging) absorption bands at $890 \mathrm{~cm}^{-1}$ and in the $730-650 \mathrm{~cm}^{-1}$ region, respectively. On the contrary, vinyl bonds are observed without any doubt (at $990 \mathrm{~cm}^{-1}$ and $909 \mathrm{~cm}^{-1}$ ), explaining why we assimilated the $1640 \mathrm{~cm}^{-1}$ band to vinyls. On the one hand, this band has a known extinction molar coefficient $\varepsilon$, and, on the other hand, we could neither use the band at 990 $\mathrm{cm}^{-1}$ because of overlapping with the one of trans-trans-dienes at $985 \mathrm{~cm}^{-1}$ nor the band at $909 \mathrm{~cm}^{-1}$ because of its overlapping with the $v_{17}\left(B_{2}\right)$ band of pristine polystyrene repetition units (see Figure S1 of the Supplementary Information).

Since all spectra are collected at $11 \mathrm{~K}$ and since $\varepsilon$ is function of the temperature, the knowledge of $\varepsilon$ at $11 \mathrm{~K}$ for the vibrations under study is mandatory. Their determination was already presented in the previous article [8]. They are nevertheless recalled in Table 3. For the allyl radical (band at $942 \mathrm{~cm}^{-1}$ ), to our best knowledge, there is no estimation of $\varepsilon$. So, instead

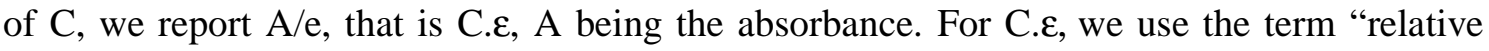
concentration" and as we express $\mathrm{e}$ in $\mathrm{g} \cdot \mathrm{cm}^{-2}$ then C.E is expressed in $\mathrm{cm}^{2} \cdot \mathrm{g}^{-1}$. All concentrations and C.E for the allyl radical case are rescaled to the aliphatic moiety fraction. We are in fact supposing that all the chemical groups followed here are formed in the aliphatic moiety.

\begin{tabular}{|c|c|c|c|}
\hline Wavenumber $\left(\mathrm{cm}^{-1}\right)$ & Attribution & $\varepsilon_{\mathrm{RT}}\left(\mathrm{L} \cdot \mathrm{mol}^{-1} \cdot \mathrm{cm}^{-1}\right)$ & $\varepsilon_{11 \mathrm{~K}}\left(\mathrm{~L} \cdot \mathrm{mol}^{-1} \cdot \mathrm{cm}^{-1}\right)$ \\
\hline 1640 & Asymmetric alkenes (vinyls) & $36[17]$ & $43[16]$ \\
985 & Trans-trans-diene & $360[18]$ & $900[16]$ \\
967 & Trans-vinylene & $169[19]$ & $260[16]$ \\
\hline
\end{tabular}

Table 3: Molar extinction coefficients $\mathcal{E}$ (in L. $\mathrm{mol}^{-1} . \mathrm{cm}^{-1}$ ) at RT and at $11 \mathrm{~K}$, for the main new double bonds formed polyethylene under irradiation. [17]: average of the values given in 
Table 7-6, page 96. [18]: determined in liquid phase from 9,11-linoleic acid in $\mathrm{CS}_{2}$. [19]: determined in solid phase from n-pentatriacontene $\left(\mathrm{C}_{35} \mathrm{H}_{70}\right)$ in n-hexatriacontane $\left(\mathrm{C}_{36} \mathrm{H}_{74}\right)$.

Depending on the chemical group considered and on the irradiation temperature, the evolution of the concentration as a function of dose can present different behaviors. If the curve presents a tendency toward saturation, equation (3) is used to fit the experimental data. If the curve is linear, equation (4) is used. Finally, for curve presenting an upward curvature, equation (5) is used.

$$
\begin{gathered}
C=P_{1}+P_{2} \cdot\left(1-\exp \left(-P_{3} \cdot D\right)\right) \\
C=P_{4}+P_{5} \cdot D \\
C=P_{6}+P_{7} \cdot D+P_{8} \cdot D^{2}
\end{gathered}
$$

In these equations, $\mathrm{D}$ is the dose in $\mathrm{Gy}$ and $\mathrm{P}_{\mathrm{i}}(\mathrm{i}=1 \ldots 8)$ are free parameters to be determined during the curve fitting. The use of $\mathrm{P}_{1}$ from (2), $\mathrm{P}_{4}$ from (3) and $\mathrm{P}_{6}$ from (4) allows a supplementary degree of freedom to $\mathrm{C}_{0}$ values.

\section{Results}

All experimental data presented in this section have been obtained from the spectra of the irradiated film, for which the corresponding spectrum of the same film, before irradiation, has been subtracted.

It can be recalled here that, at $11 \mathrm{~K}$, only excitation transfers are effective, whereas at RT, radical migration has also to be taken into account [8]. Results will be presented separately at these two temperatures, to evaluate separately the effects of excitation transfers in presence and in absence of mass transfers. Difference obtained between the results allows extracting the radical transfer ratio, assuming that excitation transfers are not temperature dependent.

It is generally considered that alkyl radicals are the first species to be formed in polymers irradiated under inert atmosphere: following this radical should have been very interesting. Nevertheless, the characteristic infrared band of this radical lies at $3017 \mathrm{~cm}^{-1}$ [16], too close to the $v_{2}^{\prime}\left(A_{1}\right)$ (see Figure S1 of the Supplementary Information) band of polystyrene at 3025 $\mathrm{cm}^{-1}$ to be exploitable. Hence, this radical will not be followed in this article.

\subsection{Trans-vinylenes}

Trans-vinylenes results and comparison between the two irradiation temperatures were the subject of the previous article [8]. Hence, the figure is not given in the main text of this 
article, but for sake of clarity, it is recalled in Figure S2 of the Supplementary Information. This band is observed whatever the irradiation temperature, and a decrease in its formation is observed at both $11 \mathrm{~K}$ and RT when the aromatic concentration increases in the copolymers. This observation implies that excitation transfers from the aliphatic to the aromatic moiety are very efficient at low temperature, but that reactive species transport, when irradiation is performed at RT, participates to the radiation stabilization too.

\subsection{Allyl radicals}

Figure 1 gathers the relative concentration evolutions (in $\mathrm{cm}^{2} / \mathrm{g}_{\text {aliphatic moiety }}$ ), as a function of dose, of allyl radicals from ethylene/styrene random copolymers and from polyethylene irradiated under vacuum; irradiations performed at $11 \mathrm{~K}$ on the left and at RT on the right.
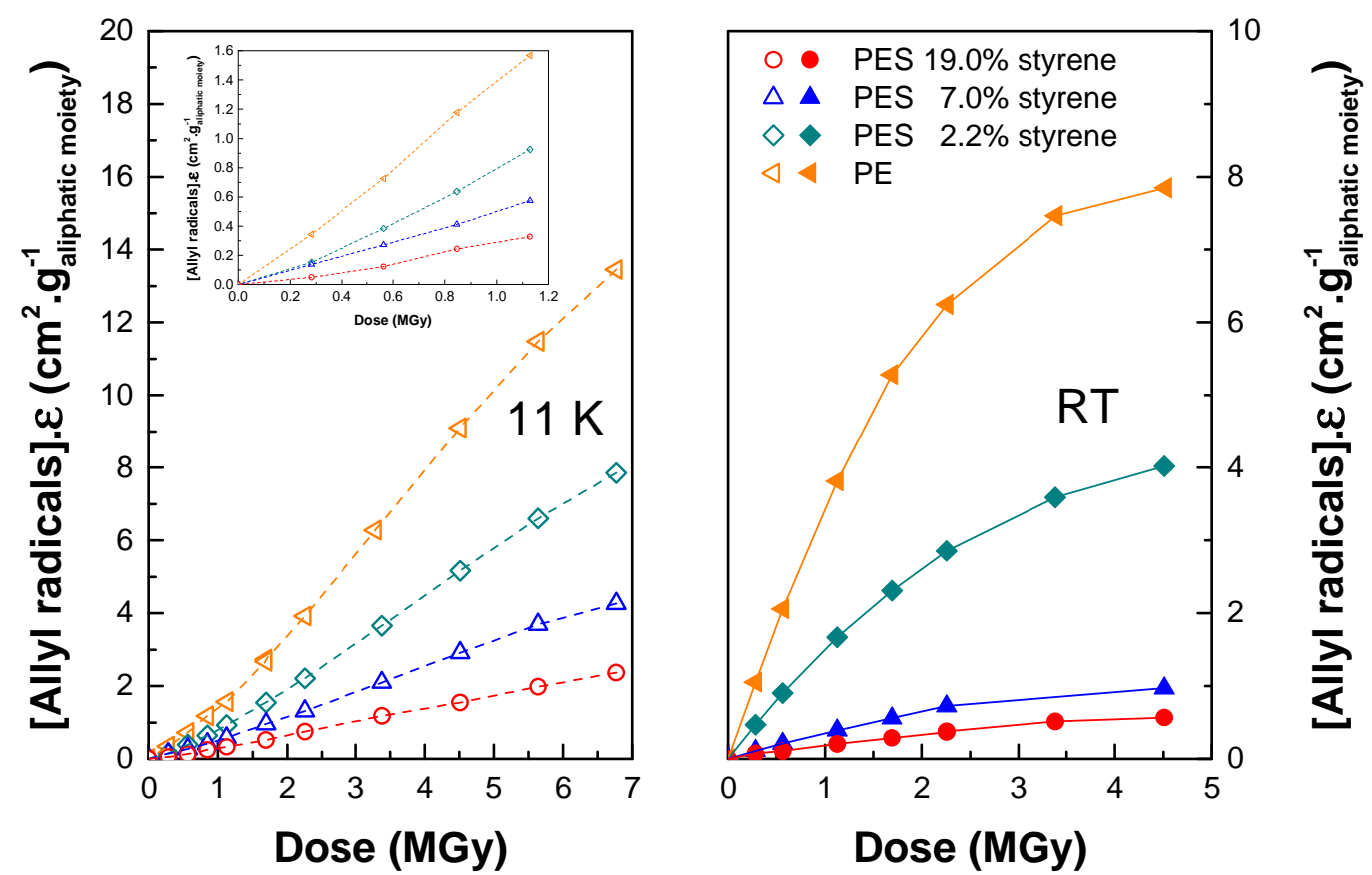

Figure 1. Allyl radical relative concentration evolutions (in $\mathrm{cm}^{2} / g_{\text {aliphatic moiety }}$ ) in the aliphatic moiety of the ethylene/styrene random copolymers, irradiated at $11 \mathrm{~K}$ on the left (insert: zoom on the low dose area) and at RT on the right. All infrared spectra are recorded at $11 \mathrm{~K}$. 
Whatever the aromatic content in the copolymer, the evolution of the concentration of allyl radicals as a function of dose presents a slight upward curvature at $11 \mathrm{~K}$ while it presents a tendency towards saturation at RT.

Moreover, for a given irradiation temperature, the presence of aromatic rings in the copolymer contributes to an important decrease of this radical concentration. In fact, whatever the dose in the dose range studied, allyl concentration decreases when styrene concentration increases in the polymer. It can be observed that at RT, the formation of this radical is almost suppressed in the case of PES $19.0 \%$ styrene. Since the decrease in the formation of this radical is effective at low temperature and low doses, it can be concluded that the radioprotection of the aliphatic component by the benzene rings is also effective at $11 \mathrm{~K}$ for this chemical group.

Moreover at $11 \mathrm{~K}$, the higher the dose, the higher the radiation protection effect is. The increase in styrene content induces a modification in the evolution of the concentration with dose: the upward curvature evolution visible for polyethylene is progressively faded by the introduction of styrene or would occur at doses higher than studied here. 


\subsection{Trans-trans-dienes}

Figure 2 gathers the relative concentration evolutions (in $\mathrm{mol} / \mathrm{kg}_{\text {aliphatic moiety }}$ ), as a function of dose, of trans-trans-dienes from ethylene/styrene random copolymers and from polyethylene irradiated under vacuum. Irradiations performed at $11 \mathrm{~K}$ and RT are on the left and right sides, respectively.
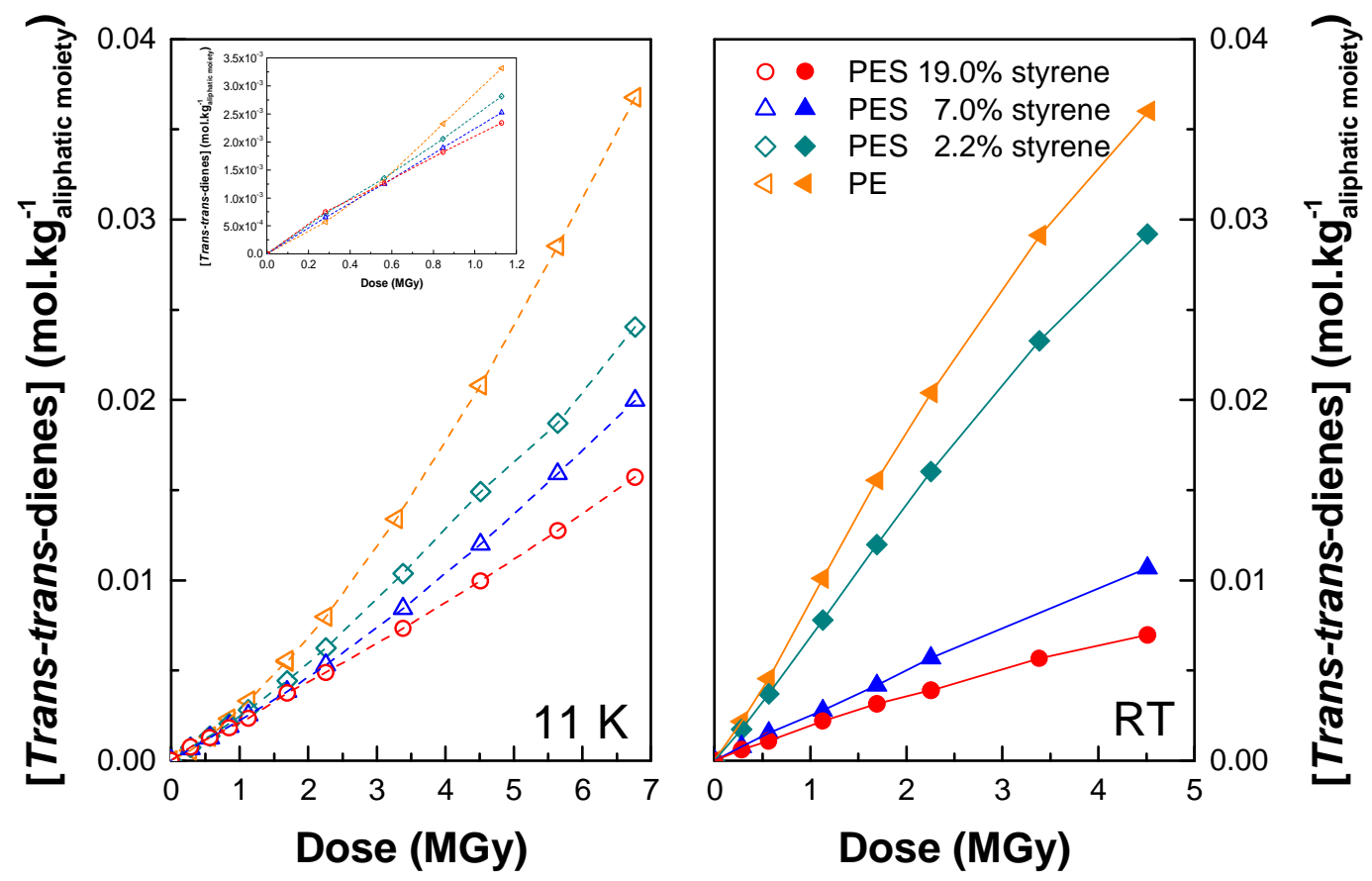

Figure 2. Trans-trans-diene concentration evolutions (in mol/ $/ g_{\text {aliphatic moiety) }}$ in the aliphatic moiety of the ethylene/styrene random copolymers, irradiated at $11 \mathrm{~K}$ on the left (insert: zoom on the low dose area) and at RT on the right. All infrared spectra are recorded at $11 \mathrm{~K}$.

Because of chemical requirements to form trans-trans-diene bonds, its creation ability in PES copolymers should statistically decrease compared to PE. In fact, the carbon linked to the benzene ring is ineffective to form this kind of bond; two successive ethyl groups are required. For instance, if styrene-ethylene-styrene sequences are present, ethyl groups concerned are no longer effective to form an unsubstituted trans-trans-diene bond. We estimated this geometric efficiency loss, by calculating the number of ethyl groups able to lead to the formation of unsubstituted trans-trans-diene bond on the total number of ethylene repetition units in the copolymer. Repartition is randomly chosen in accordance with the 
polymer synthesis, which prevents head/head and tail/tail polymerization of styrene. At the highest styrene molar percentage presented in this article, a geometric efficiency loss of less than $4 \%$ is calculated. We will thus neglect it in the following.

In pure PE, we have shown that at low temperature, low LET and low dose, trans-transdienes are not formed [20], indicating that long range radical migration is required for the formation of this chemical group. This is no more the case at the LET considered here, as initial yield is not zero (Figure 2). Hence, at this temperature that is without radical migration, and at low dose that is without pre-existing radicals, trans-trans-diene should be created close to the ion path, because of the higher probability of having two radicals very close to each other: likely an alkyl and an allyl. In PES, the aromatic rings presence has no or almost no influence on its formation at low doses. At $11 \mathrm{~K}$ but at higher doses, tracks overlap and the probability of the statistical encounter of the two radicals increases. As a result, the overall concentration evolution of trans-trans-dienes with the dose presents an upward curvature. On the other hand, when dose increases, the influence of the aromatic rings begins to be visible: the concentration of trans-trans-dienes created in the aliphatic component of the copolymers decreases when the aromatic moiety concentration in the copolymer increases. Again, the upward curvature behavior of the concentration slowly disappears when the styrene content increases.

At RT, when radicals' migration is allowed, trans-trans-dienes concentration decreases when styrene content increases. As the accepted mechanism implies the encounter of two radicals, and as the radical concentration decreases when styrene content increases (because they are less formed due to energy transfers and also because a great part of the two radicals will preferentially migrate towards styrene units), this result was expected.

\subsection{Vinyls}

Figure 3 gathers the evolution of relative concentration of vinyls (in $\mathrm{mol} / \mathrm{kg}_{\text {aliphatic moiety }}$ ) as a function of dose, from ethylene/styrene random copolymers and from polyethylene irradiated under vacuum. Irradiations performed at $11 \mathrm{~K}$ and RT are on the left and right sides, respectively. 

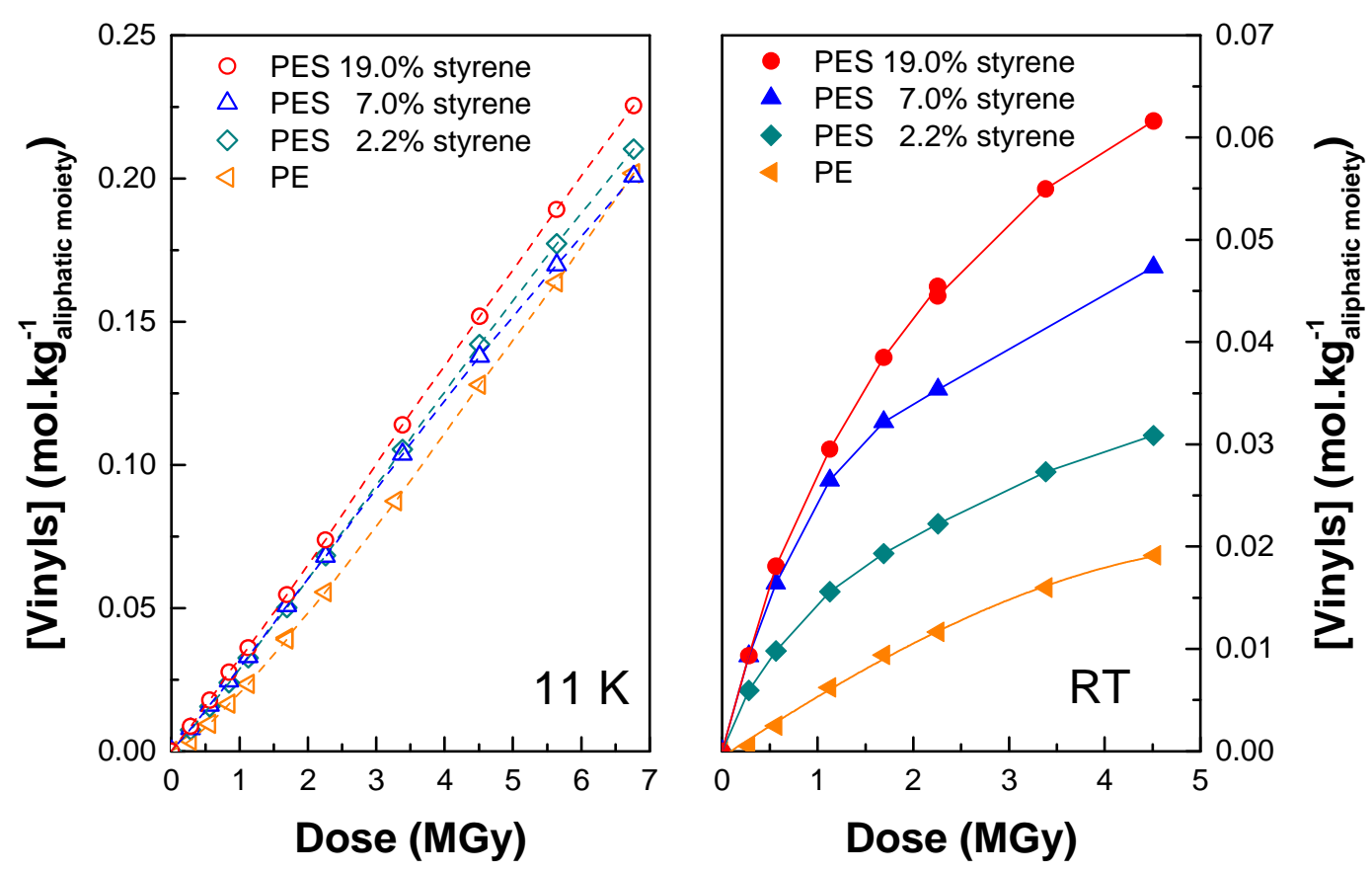

Figure 3. Vinyl concentration evolutions (in mol/kgaliphatic moiety) in the aliphatic moiety of the ethylene/styrene random copolymers, irradiated at $11 \mathrm{~K}$ on the left and at $R T$ on the right. All infrared spectra are recorded at $11 \mathrm{~K}$.

In an earlier work [21-23] and in agreement with literature [24], we have shown that vinyl formation in polyethylene requires high LET to be effective. In other words, a high density of reactive species is needed. The yields at RT were found smaller than at $11 \mathrm{~K}$. We suggest that, as an irradiation temperature of $11 \mathrm{~K}$ avoids, or at least drastically limits radical migration, the local density of reactive species should be higher at $11 \mathrm{~K}$ than at RT and this could explain the higher vinyl concentration at $11 \mathrm{~K}$.

It is observed that at $11 \mathrm{~K}$, the vinyl concentration evolution is linear with dose and it is almost not influenced by the styrene concentration. On the contrary, at RT, its concentration evolution presents a tendency towards saturation and an important influence of the styrene concentration is visible.

Whatever the dose at RT, the concentration of vinyl groups in the aliphatic moiety is higher at higher styrene concentration. If we compare copolymer PES $19.0 \%$ styrene and polyethylene, it can be concluded that the relative importance of the different defects is greatly modified by the presence of aromatic rings. Whatever the irradiation temperature, 
trans-vinylenes remain the main radiolysis defect ${ }^{\mathrm{i}}$, but at $11 \mathrm{~K}$, vinyls are no longer negligible and represent about $30 \%$ of the unsaturations, at $4.5 \mathrm{MGy}$.

The assumption we made that vinyl groups are created in the aliphatic moiety might be questioned. As we have already shown that the radiation protection effect conferred by the benzene rings is efficient but at its expense [12], it makes sense to analyze if vinyl formation comes, at least partially, from the destruction of the styrene units by energy transfers. Results obtained when irradiating polystyrene, PS, could give some insight on the chemical modifications presumably induced in the aromatic moiety of PES. The major modifications observed in irradiated PS are trans-vinylenes, vinylidenes, benzene and alkynes [25]. Vinyl creation cannot be ruled out [26] but this group is likely formed at a much lower yield. Hence, we tried to detect the formation of this chemical group in atactic polystyrene irradiated roughly in the same conditions than here $\left({ }^{18} \mathrm{O}\right.$ ions at a mean LET of $3.4 \mathrm{MeV} \cdot \mathrm{mg}^{-1} \cdot \mathrm{cm}^{2}$ irradiated at RT). Because polystyrene presents many vibration bands at wavenumbers representative of vinyls $\left(v_{5}\left(B_{2}\right)\right.$ at $980.1 \mathrm{~cm}^{-1}$ for the $990 \mathrm{~cm}^{-1}$ band and $v_{17}\left(B_{2}\right)$ at $907.0 \mathrm{~cm}^{-}$ ${ }^{1}$ for the $909 \mathrm{~cm}^{-1}$ band; see Figure S1 of the Supplementary Information), the detection sensitivity is not very good. Vinyl creation, if any, could not be quantified in the irradiated PS. Moreover, a careful inspection of the spectra of the irradiated PES did not reveal any clear presence of vinylidenes. If we would envisage that a significant part of the vinyl formation in PES is related to the destruction of the aromatic rings, this should imply a complete inversion of the ratio vinylidene/vinyl between pure PS and the aromatic moiety of PES. This is hard to conceive. Consequently, it seems wise to consider that vinyl groups are essentially located in the aliphatic moiety. Besides and irrespective of the role of energy transfers, the aromatic groups could eventually induce vinyl groups in the aliphatic moiety by beta scissions of the main chain. Under this scenario, it is expected to find a styrene group in the aromatic counterpart of the aliphatic vinyl. We could not observe, in the spectra of irradiated PES the characteristic bands $\left(1630 \mathrm{~cm}^{-1}\right.$ and $\left.1576 \mathrm{~cm}^{-1}\right)$ of these styrene groups. It makes it unlikely that the role of the aromatic content on the vinyl creation originates primarily from beta scissions.

\section{Discussion}

\footnotetext{
${ }^{\mathrm{i}}$ Contrary to what was written in our precedent article, double bonds concentration is given in
} $\mathrm{mol} / \mathrm{kg}_{\text {aliphatic moiety }}$ and not in $\mathrm{mol} / \mathrm{kg}_{\text {whole material }}$. 
The influence of the styrene concentration in the PES copolymers is function of the irradiation temperature, and, for a given temperature, is function of the chemical group formed and of the irradiation dose.

\subsection{Irradiations at $11 \mathrm{~K}$}

At $11 \mathrm{~K}$, the creation yields are dose dependent. To enable a clear discussion of the results, the defect evolution is divided in two dose categories: low doses (<1.5 MGy) and high doses (> 1.5 MGy).

\section{Low dose range}

In the low dose range and at this temperature, vinyls and trans-trans-dienes are not sensitive to the aromatic rings presence. On the contrary, allyl radicals and trans-vinylenes formation decreases when the molar styrene percentage in the copolymers increases.

Trans-vinylene bonds can be formed through three main paths [27]:

$$
\begin{gathered}
P H^{*} \rightarrow \mathrm{TV}+\mathrm{H}_{2} \\
P^{\circ}+H^{\circ} \rightarrow \mathrm{TV}+\mathrm{H}_{2} \\
P^{\circ}+P^{\circ} \rightarrow \mathrm{TV}+P_{\text {hydrogenated }}
\end{gathered}
$$

$P^{\circ}$ is an alkyl radical, TV the trans-vinylene bond. In reaction (c), $P_{\text {hydrogenated }}$ is a hydrogenated polymer. Reaction (a) is generally considered to be minor in the trans-vinylene creation process [27]. Besides, on the basis of our previous work [20], this reaction alone cannot explain the ratio of 0.5 obtained from polyethylene between radiation chemical yields at $11 \mathrm{~K}$ and at RT. Hence, the creation of trans-vinylene is assumed to happen through a sequential manner along reaction (b) or (c). At $11 \mathrm{~K}$ and at low doses, trans-vinylenes must be formed in cage, i.e. very close to creation of the initiator. As an effect of styrene concentration is observed on trans-vinylene concentration, and as radical transfer is very probably hindered at such a low temperature $\left(\mathrm{T}<\mathrm{T}_{\mathrm{g}}\right.$ for all the copolymers), reduction in the trans-vinylene creation while increasing the styrene content implies a reduction in the creation of the initial radical $\mathrm{P}^{\circ}$ through excitation transfers.

Allyl radicals are generally considered to be created through the formation or the migration of an alkyl radical in close vicinity of an alkene (trans-vinylene or vinyl). The creation of these radicals, at a temperature where radical migration is highly restricted, requires that a radical is created nearby an alkene. The decrease of the allyl yield induced be increasing aromatic content is of the order of magnitude of the one of trans-vinylenes (same 
order of magnitude but nevertheless higher by a factor of around two). This is expected as alkyl radical concentration is likely also reduced by the addition of aromatic groups.

Trans-trans-dienes are formed, at $11 \mathrm{~K}$ and at low doses, at relatively low concentration. The generally accepted mechanism is their formation by statistical encountering of an allyl radical and an alkyl radical in regions of high ionizing densities, which means very close to the ion path.

These two aforementioned mechanisms are summarized just below, with TTD representing the trans-trans-diene bond, and $\mathrm{AR}^{\circ}$ the allyl radical:

$$
\begin{gathered}
P^{\circ}+T V \rightarrow A R^{\circ} \\
P^{\circ}+A R^{\circ} \rightarrow T T D
\end{gathered}
$$

Vinyls are known to be formed in the track core or in its vicinity, and it can be observed in this work that this kind of defects are not influenced by energy transfers.

Hence, the radiation-induced defects can be separated in two categories: those which do not need a high density of ionization and for which energy transfers are efficient, like transvinylenes and allyl radicals, and those which need it and for which energy transfers are inefficient, like trans-trans-dienes and vinyls. It is deduced from our results that energy transfers are efficient in the track halo but not in the track core. Under very high excitation and ionization densities, the radiation protection of benzene rings is lost. This result is similar to results obtained on our previous work: when LET increases, i.e. when excitation and ionization density increases, the radiation protection given by the benzene ring is lost [25].

\section{High dose range}

In the high dose range, for trans-vinylene, allyl, and vinyl groups, the effect of adding styrene is substantially the same as in the low dose range. Vinyl creation is unaffected by the styrene addition whatever the dose. Trans-vinylene and allyl creation show essentially the same dependence upon styrene concentration as at low doses, even if, for the allyl radical, the slight upward curvature with dose observed for PE is progressively faded with increasing the aromatic moiety content. By contrast, the behavior of trans-trans-diene groups is remarkably different: PE shows a strong upward curvature with dose, this non-linearity being almost suppressed at the highest styrene content. An upward curvature with dose is expected for trans-trans-diene groups as their formation should occurs when a trans-vinylene is created in the vicinity of a previously created one (without any destruction process, one could guess an evolution proportional to the square of the trans-vinylene concentration). As increasing the styrene content decreases the trans-vinylene concentration, the fading of the non-linear 
behavior with dose when increasing the styrene content is not surprising. For allyl radicals, a similar trend is expected, and in fact observed, as their formation requires having an alkene in the vicinity of an alkyl radical. Why, in the allyl case, the observed magnitude is much smaller than for trans-trans-diene groups is still an open question.

\subsection{Irradiations at RT}

At room temperature, qualitatively, trans-vinylenes, allyls and trans-trans-dienes show a similar behavior as at $11 \mathrm{~K}$ : i) a downward curvature with dose, in other words a tendency towards saturation, ii) a similar influence of the aromatic content at any dose, i.e. the larger the aromatic moiety content, the lower the yields. Vinyl groups have a singular behavior: they show as the three other defects the same dose evolution (tendency towards saturation) but the effect of the aromatic content is reversed. The larger the aromatic moiety content, the higher the yields.

At RT, the occurrence of long-range radical migration has two main consequences: first, an enhancement of creation because of the recombination of radical-radical or of the reaction between radical radiation-induced chemical groups, and second an increase of destruction by radical attack. It is therefore necessary to consider, in addition to excitation transfers, a decrease in the radicals' concentration in the aliphatic moiety, because of the effective radical migration preferentially occurring towards the energy sinks, i.e. the aromatic rings. Excitation and species transfers reduce primary formation of radicals but also their destruction rate by radical migration. As the styrene content increases in the copolymer, one may expect less creation and less destruction because of radical attack.

\section{$\underline{\text { Trans-vinylenes, allyls and trans-trans-dienes }}$}

In PE, for the three defects considered here, increasing the temperature to RT induces an increase of the radiation chemical yield extrapolated at zero dose. At higher dose in the dose range studied here, the concentration of trans-vinylenes and trans-trans-dienes systematically overwhelms the concentration at $11 \mathrm{~K}$, which means that the effect of enhancement of creation compensates the enhancement of destruction.

When increasing the aromatic content, the balance between creation and destruction changes. This creation-to-destruction balance evolution differs as a function of the defect considered. As a single example, for the highest aromatic content, in the dose range studied, the concentrations of allyl radicals and of trans-trans-dienes are systematically lower at RT than at $11 \mathrm{~K}$. On the contrary, the trans-vinylenes concentrations are close, except at the 
highest dose where the RT concentration is slightly smaller. From a mechanistic point of view, comparing the results at RT and $11 \mathrm{~K}$ is not systematically straightforward. For transvinylenes presented in the preceding article [8], a direct comparison of the formation mechanism is possible because mechanisms are not different. Nevertheless, in other cases, these formation mechanisms can be completely different at $11 \mathrm{~K}$ and at RT. For instance, for trans-trans-dienes which are almost not formed at low dose at $11 \mathrm{~K}^{\mathrm{i}}$, whereas they are formed almost exclusively by radical migration at RT: direct comparison is a non-sense.

The concentration at saturation ${ }^{\mathrm{ii}}$ is seen to decrease when styrene concentration in PES increases. It can be deduced from that observation that, because of radical attack, destruction is more and more preponderant when the aromatic content increases. Moreover, the initial slope for formation of trans-trans-dienes, trans-vinylenes and allyl radicals decreases when the initial styrene molar content in PES increases. As one of the reaction path to form a transtrans-diene is the recombination of an alkyl radical and an allyl radical (reaction (e)) [16], this observation was expected. It can also be noted that the addition of aromatic rings has a greater effect on the formation of allyl radicals than on the formation of trans-vinylenes. If we hypothesize, simplistically, that allyl radicals are formed via reaction (d), then it can be supposed that the presence of styrene decreases the formation of alkyl radicals.

\section{$\underline{\text { Vinyls }}$}

Effect of the irradiation on the vinyls formation is very different from the three first defects, but also as a function of the temperature. As it was already introduced, there is no influence of the styrene content presence at $11 \mathrm{~K}$ on this chemical group. At RT, vinyl concentration increases with the styrene concentration in the PES. We have also concluded in the results section that the increase of vinyl concentration with the aromatic content is hardly related to either a creation in the aromatic moiety or a creation in the aliphatic moiety related to beta scissions. Then, the best alternative seems to consider, first, that creation is not affected by the aromatic addition and, second, that destruction much decreases when increasing the styrene concentration. As, at both temperatures, creation of vinyls is induced only in regions where a high density of radicals is created, it is not so surprising that the RT creation is not affected by the energy or species transfer towards aromatic groups. Concerning destruction, as indicated just above, with styrene addition, primary formation of radicals is

\footnotetext{
${ }^{\mathrm{i}}$ At low LET, trans-trans-dienes are not created at all at $11 \mathrm{~K}$.

ii Although the dose range is too small to speak, rigorously, of concentration at saturation, it seems that the addition of styrene greatly decreases this parameter.
} 
reduced because of excitation transfers. Moreover, it has also been evidenced that radical migration occurs preferentially towards the aromatic repetition units instead of towards the defects formed in the aliphatic moiety.

\section{Conclusion}

We have compared the formation of the different radiation-induced unsaturated groups in ethylene/styrene random copolymers, as a function of the styrene content and of the irradiation temperature. Irradiating at $11 \mathrm{~K}$ and at RT allowed us to discriminate energy and radical migration.

In copolymers, irradiation temperature influences very differently the formation of the main defects. It has been proven that at $11 \mathrm{~K}$, aromatic rings cannot protect the aliphatic repetition units which are in the track core or in its vicinity. In fact, we have shown that there is no influence of their presence on the trans-trans-diene and the vinyl concentration, these two defects needing high ionizing density to be formed. In the case of chemical groups which are created whatever the excitation and ionization density, like trans-vinylenes and allyl radicals, a decrease in their concentration is observed when the styrene content increases in PES. This is a clear indication of the excitation transfers efficiency in the track halo.

At RT, where both transfers are allowed, concentrations present an evolution which is always influenced by the aromatic rings presence. Trans-vinylenes, allyl radicals and transtrans-dienes see their concentrations decrease when the aromatic moiety content increases in the copolymer. Vinyls concentration evolution presents a behavior very different from those of the other defects: it increases with styrene content.

\section{References}

[1] Schoepfle CS, Fellows CH. Gaseous Products from Action of Cathode Rays on Hydrocarbons. Industrial \& Engineering Chemistry. 1931;23:1396-8.

[2] Alexander P, Charlesby A. Radiation protection in copolymers of isobutylene and styrene. Proceedings of the Royal Society of London Series A Mathematical and Physical Sciences. 1955;230:136-45.

[3] Basheer R, Dole M. Effects of copolymer composition on the formation of ionic species, hydrogen evolution, and free-radical reaction in $\gamma$-irradiated styrene-butadiene random and block copolymers. Journal of Polymer Science: Polymer Physics Edition. 1984;22:1313-29.

[4] Witt E. The effect of polymer composition on radiation-induced crosslinking. Journal of Polymer Science. 1959;41:507-18. 
[5] Partridge RH. Excitation Energy Transfer in Alkanes. I. Exciton Model J Chem Phys. 1970;52:2485.

[6] Partridge RH. Excitation Energy Transfer in Alkanes. II. Experimental Demonstration. J Chem Phys. 1970;52:2491.

[7] Turro NJ. Energy transfer processes. Pure and Applied Chemistry1977. p. 405.

[8] Ferry M, Bessy E, Harris H, Lutz PJ, Ramillon JM, Ngono-Ravache Y, et al. Aliphatic/Aromatic Systems under Irradiation: Influence of the Irradiation Temperature and of the Molecular Organization. The Journal of Physical Chemistry B. 2013;117:14497.

[9] Ferry M, Dannoux-Papin A, Dély N, Legand S, Durand D, Roujou JL, et al. Chemical composition effects of methylene containing polymers on gas emission under $\gamma$-irradiation. Nuclear Instruments and Methods in Physics Research Section B: Beam Interactions with Materials and Atoms. 2014;334:69-76.

[10] Ferry M, Pellizzi E, Boughattas I, Fromentin E, Dauvois V, de Combarieu G, et al. Effect of cumulated dose on hydrogen emission from polyethylene irradiated under oxidative atmosphere using gamma rays and ion beams. Radiation Physics and Chemistry. 2016;118:124-7.

[11] Ventura A, Ngono-Ravache Y, Marie H, Levavasseur-Marie D, Legay R, Dauvois V, et al. Hydrogen Emission and Macromolecular Radiation-Induced Defects in Polyethylene Irradiated under an Inert Atmosphere: The Role of Energy Transfers toward trans-Vinylene Unsaturations. The Journal of Physical Chemistry B. 2016;120:10367-80.

[12] Ferry M, Bessy E, Harris H, Lutz PJ, Ramillon JM, Ngono-Ravache Y, et al. Irradiation of Ethylene/Styrene Copolymers: Evidence of Sensitization of the Aromatic Moiety As Counterpart of the Radiation Protection Effect. The Journal of Physical Chemistry B. 2012;116:1772-6.

[13] Ferry M, Ngono-Ravache Y, Aymes-Chodur C, Clochard MC, Coqueret X, Cortella L, et al. Ionizing Radiation Effects in Polymers. Reference Module in Materials Science and Materials Engineering: Elsevier; 2016.

[14] Lee WA, Knight GJ. Ratio of the glass transition temperature to the melting point in polymers. British Polymer Journal. 1970;2:73-80.

[15] Ziegler JF, Ziegler MD, Biersack JP. SRIM - The stopping and range of ions in matter (2010). Nucl Instrum Methods Phys Res, Sect B. 2010;268:1818-23.

[16] Mélot M. Matériaux organiques irradiés à très basse température et à différents pouvoirs d'arrêt : cas du polyéthylène et des molécules de cyclohexane isolées en matrice [Spécialité Milieux denses et Matériaux]. Caen: Université de Caen; 2003.

[17] Klöpffer W. Introduction to Polymer Spectroscopy. Berlin: Springer-Verlag; 1984.

[18] Ahlers NHE, Brett RA, McTaggart NG. An infra-red study of the cis- and trans-isomers of some $\mathrm{C}_{18}$ fatty acids. Journal of Applied Chemistry. 1953;3:433-43. 
[19] Kock RJd, Hol PAHM, Bos HF. Infrared determination of unsaturated bonds in polyethylene. Fresenius J Anal Chem 1964;205:371-81.

[20] Mélot M, Ngono-Ravache Y, Balanzat E. Very low temperature irradiation of aliphatic polymers: Role of radical migration on the creation of stable groups (O-127). Nuclear Instruments and Methods in Physics Research Section B: Beam Interactions with Materials and Atoms. 2003;208:345-52.

[21] Balanzat E, Betz N, Bouffard S. Swift heavy ion modification of polymers. Nucl Instrum Methods Phys Res, Sect B. 1995;105:46.

[22] Balanzat E, Bouffard S, Moël AL, Betz N. Physico-chemical modifications induced in polymers by swift heavy ions. Nucl Instrum Methods Phys Res, Sect B. 1994;91:140.

[23] Ngono-Ravache Y, Corbin D, Gaté C, Mélot M, Balanzat E. Alkyne creation in aliphatic polymers: influence of side groups. J Phys Chem B. 2007;111:2813.

[24] Sun Y, Zhu Z, Jin Y, Liu C, Wang Z, Liu J, et al. The effects of high electronic energy loss on the chemical modification of polyimide. Nucl Instrum Methods Phys Res, Sect B. 2002;193:214.

[25] Ferry M, Ngono-Ravache Y, Picq V, Balanzat E. Irradiation of Atactic Polystyrene: Linear Energy Transfer Effects. Journal of Physical Chemistry B. 2008;112:10879.

[26] Balanzat E, Bouffard S, Bouquerel A, Devy J, Gaté C. Swift heavy ion irradiation of polystyrene. Nuclear Instruments and Methods in Physics Research Section B: Beam Interactions with Materials and Atoms. 1996;116:159-63.

[27] Tabata Y. Cross-Linking of Hydrocarbon Polymers and Their Model Compounds. Radiation Effects on Polymers: American Chemical Society; 1991. p. 31-43.

[28] Nyquist RA, Putzig CL, Leugers MA, McLachlan RD, Thill B. Comparison of the Vibrational Spectra and Assignments for $\alpha$-Syndiotactic, $\beta$-Syndiotactic, Isotactic, and Atactic Polystyrene and Toluene. Applied Spectroscopy. 1992;46:981-7.

[29] Liang CY, Krimm S. Infrared spectra of high polymers. VI. Polystyrene. Journal of Polymer Science. 1958;27:241-54. 


\section{Supplementary Information}

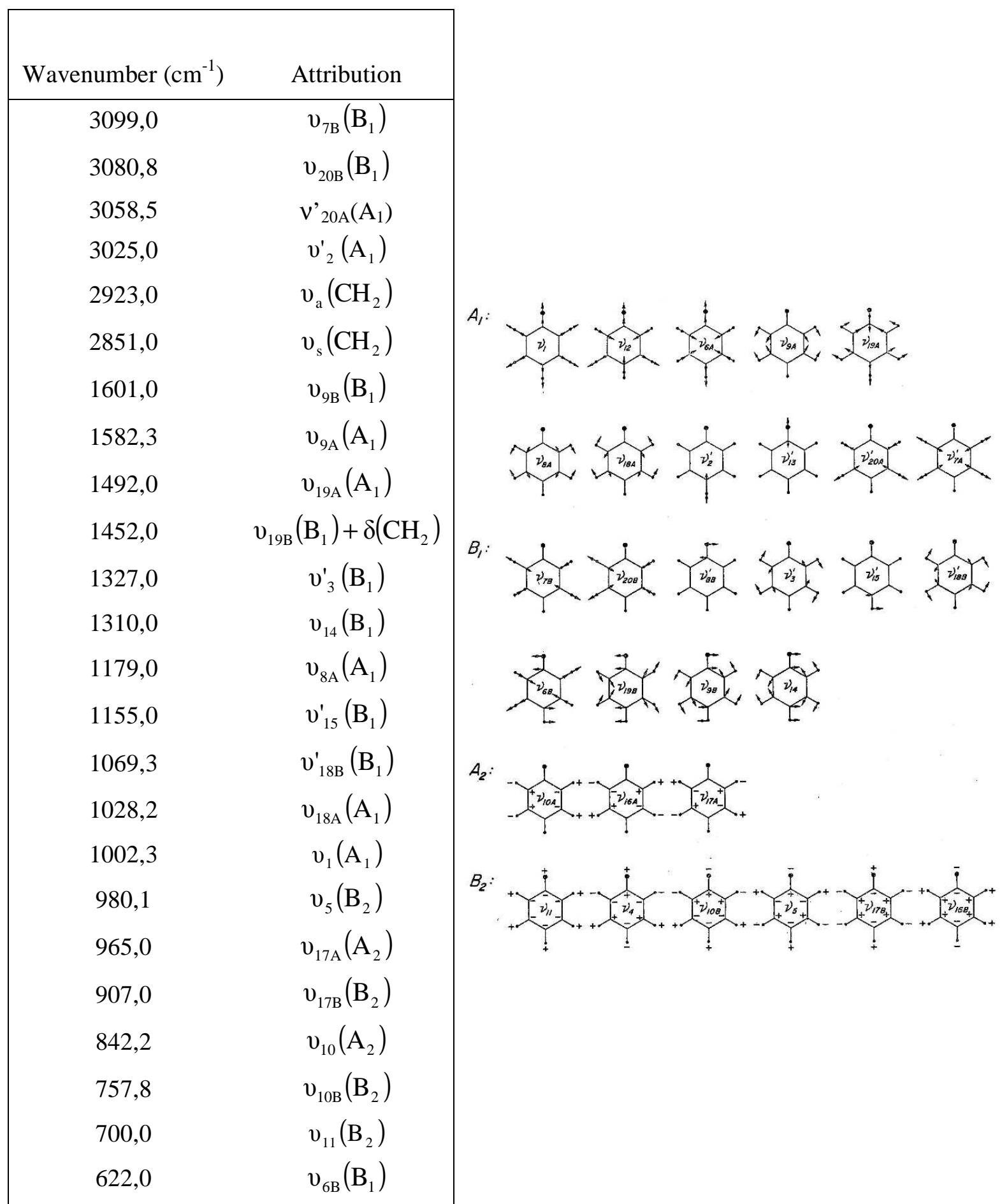

Figure S1. Polystyrene infrared band attribution [28], on the left, according to the notation of Liang and Krimm [29], on the right. 

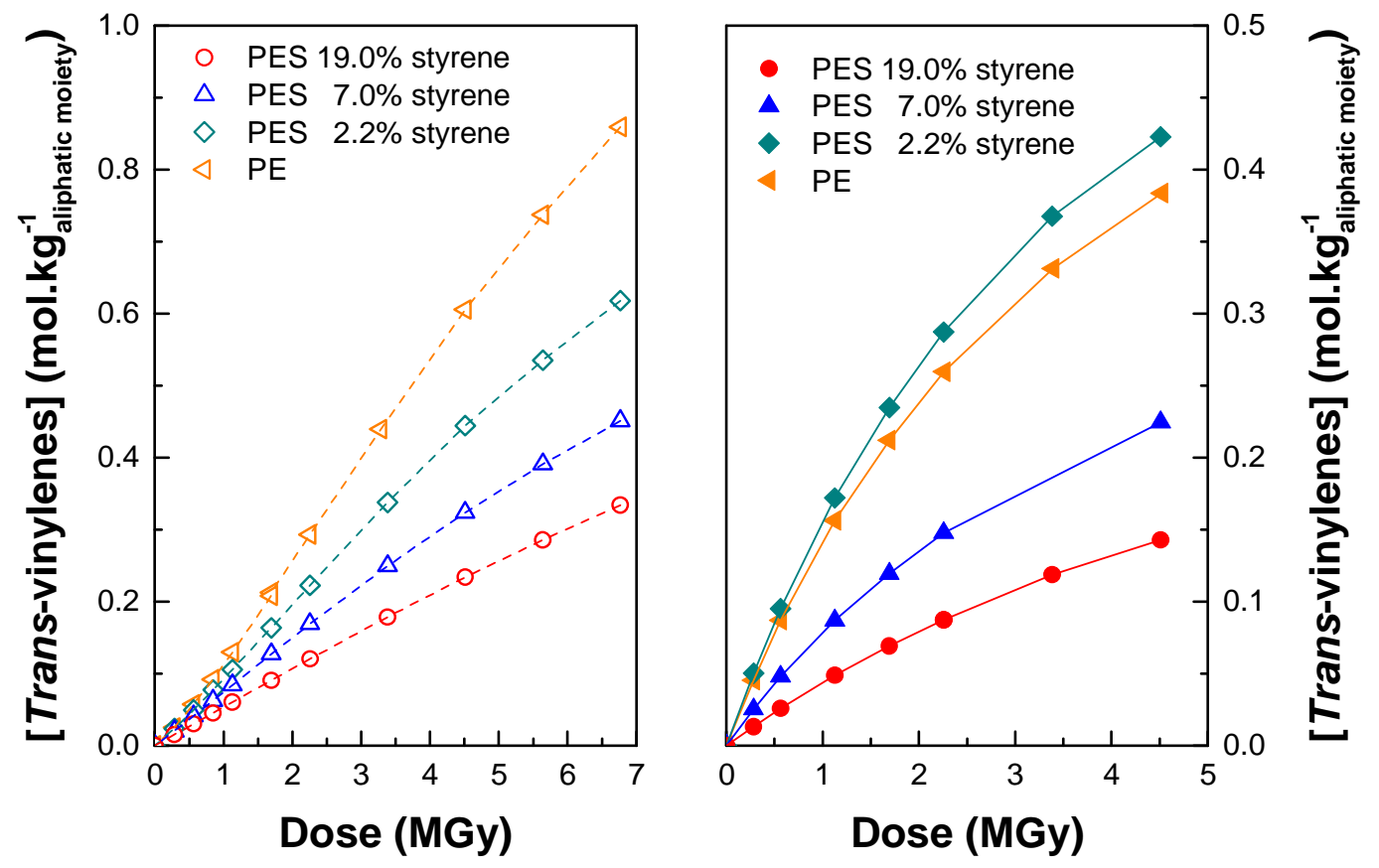

Figure S2. Trans-vinylenes concentration evolutions (in mol/kg aliphatic moiety) in the aliphatic moiety of the ethylene/styrene random copolymers, irradiated at $11 \mathrm{~K}$ on the left and at room temperature on the right. All infrared spectra are recorded at $11 \mathrm{~K}$. 